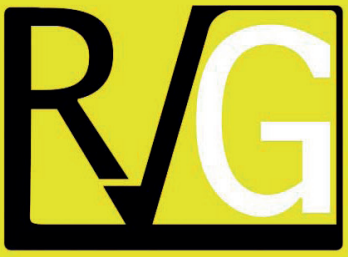

Julio - Septiembre, 2021

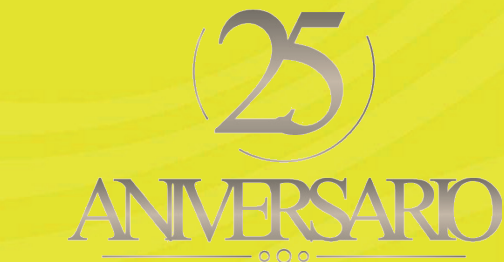

ANMERSARO
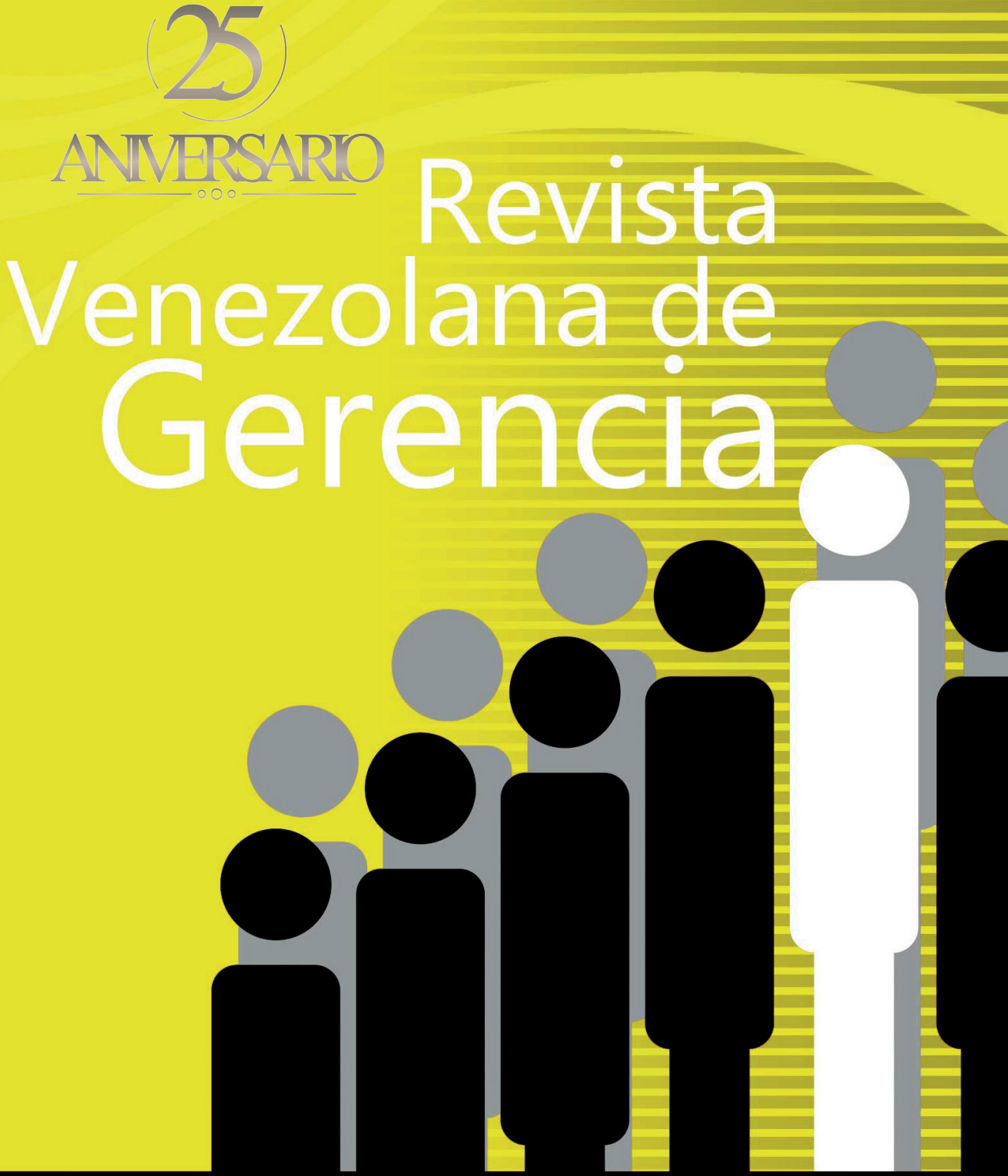

UNIVERSIDAD DEL ZULIA (LUZ)

Facultad de Ciencias Económicas y Sociales Centro de Estudios de la Empresa

ISSN 1315-99

Esta obra está bajo una licencia de Creative Comm Reconocimiento-NoComercial-Compartirlgual 3.0 Unpo http://creativecommons.org/licenses/by-nc-sa/3.0/deed.es 
COMO CITAR: Palacios, J. P., ToledoCórdova, M. F., Miranda-Aburto, E. y Flores Farro, A. (2021). Políticas públicas y gobernanza participativa local. Revista Venezolana de Gerencia, 26(95), 564-577. https://doi. org/10.52080/rvgluz.27.95.8
Universidad del Zulia (LUZ)

Revista Venezolana de Gerencia (RVG)

Año 26 No. 95 Julio-Septiembre 2021, 564-577

ISSN 1315-9984 / e-ISSN 2477-9423

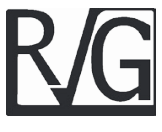

\title{
Políticas públicas y gobernanza participativa local
}

\author{
Palacios Garay, Jessica Paola* \\ Toledo-Córdova, Marianela Francisca** \\ Miranda-Aburto, Elder Jaime ${ }^{* * *}$ \\ Flores Farro, Angelica****
}

\section{Resumen}

La gobernanza se manifiesta cuando la toma de decisiones públicas proviene de un poder colectivo, es decir, conformado por las autoridades públicas, la sociedad civil y el sector privado, con igualdad en sus decisiones. El objetivo de la investigación fue analizar las políticas públicas y gobernanza participativa local desde la mirada de los colaboradores de una municipalidad de Lima, la investigación se enmarca dentro del paradigma interpretativo o naturalista; fue de enfoque cualitativo, diseño fenomenológico, teniendo como muestra 13 personas, constituida por colaboradores que laboran en la municipalidad, vecinos del distrito de Lima. El instrumento utilizado fue la entrevista semi estructurada. Los resultados indican que las políticas públicas municipales, se implementan desde de actividades puntuales, desarticuladas y desvinculadas con los instrumentos de gestión del gobierno local, asimismo no responden a los lineamientos de la política nacional definida por el gobierno peruano. Se necesita una gobernanza en función de la participación ciudadana interviniendo los actores a nivel público, privado y sociedad civil para conocer las necesidades básicas de la ciudadanía y, a partir de la elaboración de políticas públicas, produzca un impacto positivo en la población, buscando una integración vecinal.

Palabras claves: Gobernanza participativa; políticas públicas; participación ciudadana

Recibido: 11.09 .20

Aceptado: 15.12 .20

Docente de la Universidad Norbert Wiener, con Doctora en Educación y calificada como docente Investigadora Renacyt. Perú. Correo: jessica.palacios@uwiener.edu.pe. ORCID: https://orcid.org/0000-0002-2315-1683

* Maestra en Docencia y Gestión Educativa. Especialista en la Unidad de Tecnología Educativa de la DDELM, Docente universitario en la USMP, UPN. Perú. Correo Corporativo: marianela.toledo@upn.edu.pe ORCID: https://orcid.org/0000-0002-6800-947X

*** Doctor en Derecho, Maestro en Derecho Penal y Maestro en Gestión Pública. Docente investigador en la Universidad Nacional Federico Villareal, Universidad Cesar Vallejo y Universidad Privada San Juan Bautista.

Perú. E-mail: emiranda@unfv.edu.pe. https://orcid.org/0000-0003-1632-4547

*** Docente de la Universidad Nacional de Piura, Doctorado en Gestión Pública y Gobernabilidad en la Universidad César Vallejo, con amplia experiencia en el sector público. Perú. E-mail: angelicafloresfarro@ gmail.com, Orcid: https://orcid.org/0000-0003-4642-1436 


\title{
Public policies and local participatory governance
}

\begin{abstract}
Governance manifests itself when public decision-making comes from a collective power, that is, made up of public authorities, civil society and the private sector, with equality in their decisions. The objective of the research was to analyze public policies and local participatory governance from the perspective of the collaborators of a municipality of Lima, the research is framed within the interpretive or naturalistic paradigm; It was of a qualitative approach, phenomenological design, having as a sample 13 people, made up of collaborators who work in the municipality, residents of the district of Lima. The instrument used was the semi-structured interview. The results indicate that municipal public policies are implemented from specific activities, disjointed and disconnected with the management instruments of the local government, also do not respond to the guidelines of the national policy defined by the Peruvian government. Governance based on citizen participation is needed, with actors at the public, private and civil society levels intervening to meet the basic needs of citizens and, based on the development of public policies, produce a positive impact on the population, seeking a neighborhood integration.
\end{abstract}

Keywords: Participatory governance; public policies; citizen participation

\section{Introducción}

Las políticas públicas inciden en aspectos sociales, políticos, materiales y culturales que involucran lo juvenil, como parte de la estructura social; inscribiéndose en un escenario de tiempo y espacio puntual que define su peculiaridad, limitaciones y alcances, y asimismo su complejidad, diversidad y heterogeneidad. La mirada puesta en una democracia participativa ha ido ganando terreno en la última década; su relevancia en el radar político se ha manifestado desde los años sesenta y, a partir de ese momento, ha influido en las prácticas democráticas, principalmente, de América Latina.

A lo largo del tiempo, se ha dado un sentido de pertenencia, de categorización social e identidad grupal que han ayudado a la construcción de una dimensión sociopolítica, la cual se manifiesta en los procedimientos que se dan entre los gobernantes y gobernados, estableciéndose un campo de poder, de capitales simbólicos y hábitos sociopolíticos (Aguilar et al. 2015).

Como lo indica Garcia-Castillo et al. (2020), se está en presencia de una nueva propuesta política y social; por tanto, es necesario que las instituciones recuperen y asuman una correcta responsabilidad ética que mantenga el diálogo democrático entre las estructuras y las personas. El espacio destinado para la participación de los ciudadanos está abierto, especialmente, en los estados latinoamericanos. León y Figueroa 
(2019) consideran que el funcionamiento óptimo dependerá de factores que vayan más allá de una gestión institucional; pero, a su vez, indican los beneficios de la participación ciudadana en esta nueva gestión pública y política la cual busca reestructurar las instituciones de gestión púbica, así como las relaciones entre el Estado y la sociedad civil.

El factor de participación de los ciudadanos, como lo establece Vinasco (2019), es básico para que se construya una política acertada que responda a las condiciones actuales en las que se encuentra el contexto social intervenido; también se puede ver que hay otros actores que están inmersos en forma directa o indirecta como las empresas privadas, instituciones públicas y/o mixtas y la sociedad civil.

Según plantean Conejero y Poveda (2016) en la gobernanza, los autores la asocian con la toma de decisiones públicas proveniente de un poder colectivo, o sea, constituido por autoridades públicas, la sociedad civil y el sector privado. Se busca un gobierno horizontal en el que los actores de la ciudad tanto públicos como privados se interrelacionen para que la rendición de cuentas sea más clara.

Cada distrito de forma independiente y autónoma debe emitir una ordenanza que reglamente la participación de la ciudadanía dentro de su jurisdicción para luego mediante un decreto iniciar la convocatoria de participación, señalando el tema a tratar, el mecanismo de inscripción, el lugar, la hora y fecha de ejecución. Sin embargo, el cabildo abierto, es usado principalmente para rendición de cuentas de parte de algunas autoridades, con una periodicidad anual; esto conforme al artículo 119 de la Ley Orgánica de Municipalidades N²7972 de Perú, en consecuencia, la esencia de la participación ciudadana vecinal, no está siendo ejecutada de la mejor manera, ya que los vecinos conformantes de un distrito no cuentan con un escenario donde puedan ser oídos y tomados en cuenta para la toma de decisiones, el seguimiento para un control oportuno que deben tener los gobernantes desde una supervisión directa y transparente.

El estudio se justificación teóricamente esta investigación estaría relacionada a la teoría de la nueva gestión pública, resumida por García (2007) como una administración que responde a las verdaderas demandas de la población al menor costo posible, a través de la implementación de dispositivos de control que garanticen la transparencia en los procesos, planes y resultados, permitiendo promover la participación ciudadana.

Desde el aspecto práctico este trabajo pretende lograr, analizar el impacto que tuvieron las políticas públicas implementadas desde la municipalidad, como se gestionaron las principales demandas juveniles de los jóvenes del dicho distrito en el 2019. Lo cual va permitir reflexionar respecto al desafío y reto que significa establecer políticas públicas, acorde con las características, necesidades y diversidades de los ciudadanos. La participación e intervención de los actores en el proceso de mejora, permite una apropiación del proyecto, donde los ciudadanos se involucran directamente convirtiéndose en el principal defensor de su proyecto, Traba, Barletta \& Velasquez (2020), asimismo, la modernización de la administración pública involucra la necesidad de incluir a los ciudadanos para conseguir los fines de mejora esperados, para lo cual la institución debe diseñar e implementar 
mecanismos de participación ciudadana (De la Garza y Barredo, 2018).

El estudio cuenta con una justificación legal teniendo como base la Constitución Política del Perú, la Ley de Reforma Constitucional 27680/2002 de 7 de marzo, la Ley Orgánica de Municipalidades 27972/2003 de 27 de mayo, la Ley de Bases de la Descentralización 27783/2002 de 17 de julio, la Ley de Transferencia y Acceso a la Información 27806/2002 de 2 de agosto, y la Ley Marco de Modernización de la Gestión Del Estado 27658/2002 de 16 de enero.

Desde la perspectiva metodológica, la investigación se cataloga de analítica, en el sentido que se proyectó un análisis inductivo para describir y comprender de manera clara el entorno estudiado (González-Monteagudo, 2001). Es de orden cualitativa, porque buscó el entendimiento de fenómenos explorados desde el punto de vista de los participantes (Hernández-Sampieri y Mendoza, 2018).

El diseño está referido a la fenomenología como corriente filosófica, cuya característica principal se centra en la experiencia personal, teniendo cuatro conceptos claves (Peña y Bayardo, 2016): la temporalidad (el tiempo vivido), la espacialidad (el espacio vivido), la corporalidad (el cuerpo vivido) y la relacionalidad o la comunidad (la relación humana vivida), debido a que se considera que las personas están vinculándose con el mundo y siempre enfatizan en su experiencia vivida, en relación con objetos, personas, sucesos y situaciones.

Es decir, desde el paradigma interpretativo conduce a describir e interpretar lo esencial de lo vivido, reconociendo el significado y la importancia de las ciencias sociales según la experiencia recogida (Fuster, 2019). Según plantean Ceolin et al. (2017), la realidad consiste en las experiencias subjetivas de las personas del mundo externo.

Para esta investigación, la unidad de análisis estuvo conformada por 10 colaboradores de la municipalidad, 3 vecinos del distrito de Lima. Como instrumento de recolección de datos, se elaboró una entrevista semiestructurada que, según Martínez (2006), se realiza de manera coloquial, tomando en cuenta la naturaleza específica de los entrevistados y la peculiaridad de la investigación.

Considerando el escenario actual del aislamiento social por la Pandemia sanitaria del Covid-19, los datos fueron recopilados, haciendo uso de medios electrónicos, desde el lugar domiciliario mediante entrevistas semiestructurada, a través de la vía virtual, mediante llamadas telefónicas, correos, aplicativo de WhatsApp y los entornos virtuales durante el año 2020

\section{Políticas públicas: algunas ideas}

Las políticas públicas, según plantea Pérez (2019), refieren al conjunto de acciones planificadas, ejecutadas y evaluadas por actores públicos a parir de la visualización, recopilación y análisis de las demandas sociales. Están compuestas de planes, programas y proyectos que tienen como fin disminuir o eliminar los problemas sociales y en la actualidad representan un fenómeno que afecta al estado y administración de recursos (Mora, 2002). Representan el conjunto de acciones intencionales y causales, orientadas a la realización de un objetivo de interés/beneficio público, cuyos lineamientos de acción, agentes, 
Palacios Garay, Jessica Paola; Toledo-Córdova, Marianela Francisca; Miranda-Aburto, Elder Jaime; Flores Farro, Angélica

Políticas públicas para una gobernanza participativa

instrumentos, procedimientos y recursos se reproducen en el tiempo de manera constante y coherente (Quinteros, 2020).

Las políticas públicas se encuentran sustentadas en teorias que orientan el logro de procesos de transformación social (Vásquez, 2016), por lo que científicos, políticos, consultores y analistas contribuyen en su formulación a su vez, constituye un amparo antes a los derechos fundamentales y sociales de la población (Cáceres y Quevedo, 2021). De este recorrido se deduce que la política pública se ha entendido como el medio empleado por el Estado para dar respuesta a las demandas sociales.

Por su parte, Jaime et al. (2013), mencionan que dentro de los enfoques teóricos más importantes se encuentran, el de las redes de política, el cual manifiesta que estas nacen de la interrelación entre los actores gubernamentales, sociales, políticos y económicos. En este sentido, el estado deja de ser visto como un ente vertical en la elaboración e implementación de las políticas públicas.

Este enfoque menciona una nueva forma de gobernar, donde se hace hincapié en la coordinación horizontal entre los actores públicos y privados involucrados en las problemáticas de interés común.

Wilson (2018) manifestó que hablar de políticas públicas implica centrar la atención en aquella labor primordial, podríamos decir- que lleva a cabo todo Estado o gobierno en pos de concretar alguna transformación social y su desarrollo propicia la consolidación de nuevos preceptos legales (Santamaría, Moreno y Santamaría, 2016). Tal es su relevancia que, según lo expresado por los autores, ese cúmulo de acciones que se ponen en marcha tiene como finalidad atender y/o dar solución a las demandas requeridas por un sector de la ciudadanía. En función de lo anterior, se puede asumir que las políticas públicas son acciones que ejecuta el gobierno en los diferentes ámbitos de su actividad y ante un determinado problema o reclamo de la sociedad.

\section{Participación ciudadana en el Perú}

En el Perú, tal como lo indica Alcalde (2019), se han desarrollado experiencias de participación que datan de 1980; luego del gobierno militar en 1984, se promulgó la Ley Orgánica de Municipalidades. Asimismo, se dio algunos casos de gestión en zonas como (Moquegua), Villa El Salvador (Lima), San Marcos (Cajamarca), entre otras y, en varios casos, estas experiencias se prolongaron hasta finales de los años noventa.

Actualmente, como lo menciona Aspíllaga (2019), se tiene el Acuerdo Nacional que es una herramienta de concertación y de diálogo entre el Gobierno, los partidos políticos con representación en el Congreso de la República y organizaciones representativas de la sociedad civil a nivel nacional para tener una idea más clara como país.

Es necesario que se anticipe y se vayan construyendo escenarios para la democracia, con la necesidad de que estas acciones prospectivas sean inclusivas y participativas. Para lograrlo, es importante la gobernanza como un instrumento que facilite el gobernar en apertura, en donde todos los actores se interrelacionen ante una necesidad y no solo ante una decisión.

La participación ciudadana, de acuerdo con Sánchez (2015), es el proceso de construcción social de las 
políticas públicas que responde a los intereses de una sociedad democrática. Permite que se construya un espacio público, recuperando lo público para el ejercicio ciudadano, involucrando a organizaciones de la sociedad civil en el diseño e implementación de las políticas públicas que les concierne, siendo también la forma en que los ciudadanos pueden influir en las decisiones de los gobiernos de manera directa o indirecta.

Ampliando el concepto de participación ciudadana, se plantea que esta contiene categorías, que pueden ser establecidas de acuerdo con la visión de Reyes y Ortiz, (2017) para establecer cuatro formas de relacionamiento entre la administración y la comunidad con la participación:

a) Participación-formalidad: requisito de ley que es preciso llenar, también llamada participación "sin alas", pues no pone en cuestión la lógica y estructuras de poder vigentes,

b) La Participación-integracióncooptación: las autoridades locales promueven en forma efectiva la participación, creando nuevas instancias de intervención ciudadana y manejan permanentemente el discurso de la participación no tanto para que la gente gane autonomía y capacidad de interlocución con el Estado, sino, por el contrario, para que acoja las orientaciones de los grupos políticos dominantes,

c) La Participación-concertación: el acento está en la construcción colectiva de acuerdos en torno a objetivos comunes y a los medios para alcanzarlos. Los ejes aquí son el pluralismo y la intervención directa de la ciudadanía en el análisis de su entorno y en la toma de decisiones acerca de cómo actuar para conseguir objetivos compartidos.

d) La Participación-modo de vida: la participación no se concibe como una actividad adicional a la rutina diaria de la gente, como algo que exige esfuerzos adicionales y costos, sino como valor y una norma social interiorizados desde la infancia.

\section{Gobernanza en el Perú}

De acuerdo con Pinto, Martins y Leonel (2018), la participación social se relaciona con la gobernanza; siendo necesaria la incorporación de la participación social en las acciones de los gobiernos locales para producir modificaciones en las dinámicas de gobernar, especialmente, teniendo en cuenta la exclusión social y las relaciones entre grupos sociales, que son dos organizaciones que interrelacionan las dimensiones económicas, culturales y políticas que se articulan con la gobernanza y que son capaces de promover innovaciones políticas.

La gobernanza, según Quintero (2017), refiere al grupo que conforma la sociedad y determina su forma de convivir, donde lo importante no es el gobierno en sí, sino que las autoridades van a trascender, a partir de las acciones que realizan, en los actores sociales y económicos. La gobernanza, se trata de buscar una forma más horizontal de gobernar con interrelaciones entre los diferentes actores que intervienen en la ciudad, cuyo resultado sea el bien común de los ciudadanos; por otro lado, no se ha tocado el tema sobre gobernanza participativa local.

Cuando se habla de gobernanza participativa local, en la actualidad, se observa que están incorporándose nuevos campos de acción, por ejemplo, en el escenario argentino, en donde se busca tratar de cambiar la estructura 
Palacios Garay, Jessica Paola; Toledo-Córdova, Marianela Francisca; Miranda-Aburto, Elder Jaime; Flores Farro, Angélica

Políticas públicas para una gobernanza participativa

tradicional de las organizaciones estatales que cuentan con restricciones normativas y presupuestarias para que puedan desarrollar políticas propias; por eso, están implementando el concepto de gobernanza local generando mecanismos entre instituciones que articulen un modelo único de gobernanza (Foglia y Rofman, 2019).

Para Brower (2016), gobernanza es un estilo nuevo que tienen los gobiernos y que permite crecer la cooperación e interacción entre las personas que forman parte de la sociedad. Esta cooperación e interración lo que busca es garantizar que se articule una nueva forma de orden social. Asimismo, las políticas públicas son decisiones gubernamentales que cuentan con conformidad de los sectores de la sociedad, además tienen el propósito de mitigar y resolver los problemas sociales (Rodríguez, 2017).

En lo que refiere a la gobernanza participativa local, a diferencia del Gobierno, hoy en día se está en frente a una gobernanza, donde la sociedad civil trasciende al Estado para ejercer la labor común y articulada que permite lograr objetivos comunes en la que participan actores públicos y privados en los niveles locales, regionales y nacionales (McLaverty, 2017).

Se destacan tres desafíos que tienen el gobierno a fin de instituir una gobernanza participativa local : la ausencia de un consenso popular o élite dando lugar a la participación ciudadana directa y el alcance, los poderes limitados de las innovaciones participativas y la ausencia de un liderazgo sistemático (Fung, 2015). La transparencia y las iniciativas del gobierno abierto se han convertido en vehículos para incrementar la legitimidad y la confianza en los ciudadanos, ya que ha permitido mejorar la mediación de los ciudadanos y frenar la corrupción y mala gestión (Da Cruz, et al. 2016).

Lo anterior, se asocia con la gobernanza social, la cual según Durán, García y Parra (2017), es la convivencia ciudadana como la relación que se da entre los miembros de un establecimiento que inciden en su desarrollo ético, social, afectivo e intelectual. Este concepto busca que los miembros sociales tengan responsabilidad sin excepción, buscando calidad en la formación de valores, influyendo para desarrollar habilidades sociales.

Teniendo en cuenta a Subirats (2012), la transformación social se entiende como la forma de reforzar las experiencias comunitarias en los procedimientos de formular y poner en práctica de políticas públicas, así como en articular las plataformas que van a vincular el marco local que va a experimentar entre sí, además permite la fertilización cruzada y reflexionar sobre prácticas que se van a llevar a cabo en diversos lugares, buscando recuperar el sentido político y transformador de muchas experiencias sociales.

A juicio de Pulgarín et al. (2019), el interés público implica que debe prevalecer el interés general y abdicar el absolutismo para eliminar el poder que se ha concentrado. Con esto, se busca tener un orden social o colectivo con el propósito de alcanzar objetivos elaborados y aprobados de manera colectiva. Citando a Retamozo (2009), las demandas sociales son el ejercicio de identificar una situación como injusta mediante pedidos o reclamos. Para construir una demanda, se necesitan tres registros, petición y reclamo, deseo y reconocimiento, además supone una carga subjetiva fundamental para poder identificar una relación social o particular 
que sea factible de que se lleve a un espacio público.

De acuerdo con Craviotti (2006), la concertación con la sociedad se refiere a producir consensos para reconstruir identidades sociales en las organizaciones de los sectores sociales. Concertar implica, en los hechos, establecer relaciones estables de intercambio político, coexistiendo aspectos para cooperar y generar conflictos. Los mecanismos que se utilizan para la concertación social prevén o amortiguan conflictos; no se debe entender como nuevos arreglos para reconstruir algún agente social $o$ una gestión pública en desarrollo.

\section{Gobernanza participativa en las políticas públicas en las ciudades: Resultados y discusión}

En esta sección, se determina la importancia de asumir una gobernanza participativa local activa que involucre a todos los autores que participan tanto del nivel público como político, así como a la sociedad civil, esto con el propósito de que las políticas públicas que se elaboren estén en función de los ciudadanos y para los ciudadanos, para producir un impacto positivo en ellos y que se sientan representados por sus autoridades, buscando el bien común de ellos.

Es necesario que participe la población en asuntos públicos porque a través de sus manifestaciones se puede impulsar las propuestas, ejecución y evaluación de las políticas públicas teniendo en cuenta también el interés público de la ciudadanía, con lo cual se puede manifestar una transformación social que satisfaga las necesidades de la población.

Los resultados derivados de las entrevistas realizadas, refieren que es importante que se dé una gobernanza a partir de la participación de los actores tanto a nivel político, privado y sociedad civil con el objetivo de que se conozcan las necesidades de los ciudadanos, buscando el bien común de los habitantes.

Lo expresado por los participantes coincide con Brower (2016), quien define la gobernanza como un estilo nuevo que tienen los gobiernos y que permite que crezca la cooperación e interacción entre las personas que forman parte de la sociedad. Esta cooperación e interacción busca es garantizar que se articule una nueva forma de orden social. Asimismo, Rodríguez (2017) menciona que las políticas públicas son decisiones gubernamentales que cuentan con conformidad de los sectores de la sociedad, cuyo propósito es mitigar y resolver los problemas sociales.

Por otro lado, resultó importante que la elaboración de políticas públicas se dé en función de los ciudadanos, y para los ciudadanos con el propósito de producir un impacto positivo; con ello se puede lograr una integración vecinal para transformar los procesos con gran autonomía y con una correcta capacidad de gasto. De acuerdo con Craviotti (2006), la concertación con la sociedad se refiere a producir consensos para reconstruir identidades sociales en las organizaciones de los sectores sociales.

Concertar implica, en los hechos, establecer relaciones estables de intercambio político, coexistiendo aspectos para cooperar y generar conflictos. Los mecanismos que se utilizan para la concertación social prevén o amortiguan conflictos; por tanto, no se deben ver como nuevos arreglos 
para reconstruir algún agente social o una gestión pública en desarrollo.

Otro hallazgo representativo, relacionado con la convivencia ciudadana para lograr una gobernanza participativa local, permiten afirmar que el concepto de convivencia ciudadana se relaciona con la comunicación horizontal, de esta manera, se logre una interrelación democrática entre los diversos niveles de gobierno con el propósito de que se institucionalicen las organizaciones y que se muestre el interés para obtener un marco de igualdad en las propuestas que se planteen para la ciudad.

Al respecto refieren Durán et al. (2017), que la convivencia ciudadana es la relación entre los miembros de un establecimiento que inciden en su desarrollo ético, social, afectivo e intelectual. Este concepto busca que los miembros sociales tengan responsabilidad sin excepción en la formación de valores y en el desarrollo de habilidades sociales.

En cuanto a la actuación de los gobiernos locales respecto al manejo gubernamental en el distrito, los participantes manifestaron que se debe mejorar en torno al manejo gubernamental; existe mucho distanciamiento con los ciudadanos; las autoridades en muchos casos solo ven los intereses políticos, por tanto, la población no se siente representada. Aspectos estos que se están en sintonía con lo planteado por Collado (2018), quien señala lo esencial de aumentar la participación ciudadana en el diseño de las medidas participativas y que esta sea vinculante. Sobre este orden de ideas, es importante destacar que para evitar que este paso genere clientelismo, deben realizarse capacitaciones de forma inclusiva y extensiva; crear espacios participativos donde los representantes elijan de forma democrática, citando a los sectores que conforman la sociedad civil presentando su candidatura.

Al hacer referencia a la participación de los ciudadanos y la forma como esta influye en una gobernanza participativa local; los resultados demuestran que la participación ciudadana es gravitante; integra a las autoridades gubernamentales con la sociedad civil de manera positiva con el propósito de que se dé un compromiso entre el gobierno a todo nivel y la ciudadanía, respetando su identidad y autonomía.

A juicio de Pulgarín, Bustamante y Zapata (2019), la prevalencia del interés general para eliminar el poder que se ha concentrado. Con esto, se busca tener un orden social o colectivo con objetivos elaborados y aprobados de manera colectiva.

La participación ciudadana en una gobernanza participativa, se determina que la gobernabilidad se tiene que enfocar en una buena comunicación horizontal para que la interrelación sea viable entre las diversas niveles de gobierno, el sector privado y la sociedad civil; con esto, se busque una convivencia ciudadana que tenga objetivos comunes respecto a la institucionalidad de las organizaciones y que se muestre el interés para tener un marco de igualdad en las propuestas que se planteen para la ciudad.

La transformación social experimentada en las ciudades a partir de la ejecución de políticas públicas, es señal de la cooperación de la población en los asuntos públicos, aspecto crucial pues, es a través de sus manifestaciones que se impulsan las propuestas, ejecución y evaluación de las políticas públicas que van a ser emitidas por las autoridades.

Los resultados de la investigación 
se refuerzan con lo citado por Retamozo (2009), quien define las demandas sociales como resultado de identificar una situación como injusta, se evidencian mediante pedidos o reclamos, luego se interpela y lucha por ser reconocidas. Para construir una demanda, se necesita tres registros, petición y reclamo, deseo y reconocimiento, y esto supone que se involucre subjetivamente ya que su papel es fundamental para poder identificar una relación social o particular que sea factible de que se lleve a un espacio público.

Asimismo, según plantean los participantes de la encuesta, se deberían elaborar políticas públicas en función de las demandas sociales surgidas por la ciudadanía. Aspecto importante dentro de una sociedad, porque son quienes organizarán las decisiones que tomen las autoridades en beneficio de la ciudad buscando canalizar las alternativas de solución.

Al respecto, afirma Sánchez (2015), que cuando se manifiesta sobre participación ciudadana, la define como aquel proceso de construcción social de las políticas públicas que responden a los intereses de una sociedad democrática. Entonces, la participación ciudadana permite que se construya un espacio público, recuperando lo público para el ejercicio ciudadano, involucrando a organizaciones de la sociedad civil en el diseño e implementación de las políticas públicas que les concierne.

También es el conjunto de procedimientos mediante el cual los ciudadanos puedan influir en las decisiones de los gobiernos de manera directa 0 indirecta. Asimismo, las demandas sociales son herramientas de la sociedad para organizar las decisiones que tomen las autoridades en beneficio de la ciudad para solucionar las necesidades de la población y que, a su vez, garanticen la participación de la ciudadanía en el entorno público.

Finalmente, la concertación social se debe usar como un instrumento que se debe practicar para que con la experiencia y conocimiento político se busque elaborar políticas públicas que respondan a las necesidades de la ciudadanía. Una política pública se compone de un conjunto de decisiones y acciones dirigidas a resolver un problema público (Cejudo y Michel, 2016).

La solución dependerá no sólo de la idoneidad del diseño de la política pública, sino de su eficaz implementación. Este par de ideas resumen buena parte de la literatura sobre políticas públicas y revelan uno de los atributos centrales del análisis de políticas públicas y su concreción.

Para García (2007), la nueva gestión pública apunta a la generación de una administración eficiente y eficaz, que pueda satisfacer las principales necesidades de la población, a través de mecanismos que permitan la elección de los usuarios y garanticen servicios de calidad. Para lo cual es importante la implementación de un sistema de control, que garantice la transparencia de los procesos y resultados, promoviendo la participación ciudadana.

\section{Conclusiones}

Debe existir una gobernanza en función de la participación ciudadana con la intervención de los actores de la ciudad tanto a nivel público, privado y la sociedad civil para que se conozcan las necesidades básicas de la ciudadanía y que, a partir de la elaboración de políticas públicas, se pueda producir un impacto positivo en la población, buscando una integración vecinal. La población no 
Palacios Garay, Jessica Paola; Toledo-Córdova, Marianela Francisca; Miranda-Aburto, Elder Jaime; Flores Farro, Angélica

Políticas públicas para una gobernanza participativa

tiene conocimiento sobre lo que es gobernanza, menos aún el compromiso que debe asumir en la dinámica pública y su participación en el distrito.

Se debe buscar una convivencia ciudadana, con una comunicación horizontal para que se relacione de manera democrática con los diversos niveles del gobierno, con el fin de mejorar el manejo gubernamental porque solo se basan en intereses políticos. Tener en cuenta que las demandas sociales son instrumentos que van a servir para que las autoridades tomen decisiones y elaboren políticas públicas con soluciones a las necesidades de la ciudadanía.

No existe una cultura de participación ciudadana, los vecinos no cuentan con una motivación para ser parte de todo el proceso que refiere, puesto que las acciones que se han venido dando en el desarrollo del cabildo abierto han sido reducidas a informar solo el tema mas no, todo lo que conlleva, siendo los vecinos actores que desconocen de los pasos o procedimientos que se sigue.

Las políticas públicas municipales vienen siendo implementadas desde de actividades, puntuales, desarticuladas, desvinculadas que no están articuladas a los principales instrumentos de gestión del gobierno local, asimismo no responden a los lineamientos de la política nacional de juventud. Asimismo la promoción de la participación de la ciudadanía en la toma de decisiones para la implementación de políticas municipales es un factor estratégico importante que permitirá dar atención real a las principales demandas de los pobladores del distrito.

Es por ello importante la participación de la ciudadanía en la toma de decisiones para la implementación de políticas públicas municipales es un factor estratégico importante que permitirá dar atención real a las principales demandas de los pobladores del distrito. Asimismo las políticas públicas municipales no han tenido un continuismo por la inoperancia de los gobernantes de turno, la demagogia, la corrupción y el incumplimiento de los programas trazados a mediano y largo plazo.

\section{Referencias bibliográficas}

Aguilar, J., Carreón, J., García, C., Hernández, J. y Rosas, F. (2015). Gobernanza de actitudes sociopolíticas. Revista perspectivas rurales nueva época, (27). https:// www.revistas.una.ac.cr/index. php/perspectivasrurales/article/ view/7612/8035

Alcalde, V. (2019). Prospectiva, participación e inclusión en las políticas públicas, el papel de los talleres de escenarios futuros (TEF). Revista investigaciones sociales, 22(40), 223-236. https://doi. org/10.15381/is.v22i40.15898

Aspíllaga, M. (2019). El acuerdo nacional $y$ el enfoque de la familia en las políticas públicas. Revista de investigación de la facultad de derecho, 1(1), 97-113. https://dialnet.unirioja.es/servlet/ articulo?codigo $=7261899$

Brower, J. (2016). En torno al sentido de gobernabilidad y gobernanza: delimitación y alcances. Revista internacional de Filosofía Daimon, (67), 149-162. https://doi. org/10.6018/202011

Cáceres López, R., y Quevedo, G. (2021). Régimen, derechos fundamentales y sociales en Latinoamérica,2019. Telos: revista de Estudios Interdisciplinarios en 
Ciencias Sociales, 23(1), pp. 51-66. https://doi.org/10.36390/telos231.05

Cejudo, G. y Michel, C. (2016). Coherencia y políticas públicas: Metas, instrumentos y poblaciones objetivo. Gest. polít. pública, 25(1), 3-31. https://bit.ly/2Tjej8A

Ceolin, S., Piriz, M. A., Mendieta, M. C., Siles, J., Gonzalez, J. y Heck, R. M. (2017). Elements of the sociocritical paradigm in nursing care practices: an integrative review. Revista Esc Enferm USP, 51(2), 1-25. http://dx.doi.org/10.1590/ $\underline{\mathrm{S} 1980220 \times 2016037003267}$

Collado, A. (2018). Las políticas públicas de participación ciudadana en Chile. ¿Poder ciudadano o mantenimiento de la hegemonía? Rev. Est. de Políticas Publicas, 4(1), 79-98. https://ultimadecada.uchile.cl/index. php/REPP/article/view/50424/56242

Conejero, P. y Poveda, R. (2016). Gobernanza local y políticas patrimoniales: El caso de Orihuela. Revista de estudios de la administración local y autonómica, 138-152. http://dx.doi. org/10.24965/10.24965/reala. v0i5.10348

Craviotti, C. (2006). Concertación social y territorio. Revista interacoes, 8(13). https://doi.org/10.1590/S151870122006000200004

Da Cruz, N. F., Tavares, A. F., Marques, R. C., Jorge, S., \& de Sousa, L. (2016). Measuring local government transparency. Public Management Review, 18(6), 866-893. https://doi.or $\mathrm{g} / 10.1080 / 14719037.2015 .1051572$

De la Garza, D., y Barredo, D. (2018). Redes sociales, participación ciudadana y medios alternativos: la crisis de los medios masivos de comunicación en México durante el sexenio de Enrique Peña Nieto. La opinión pública y la comunicación política en entornos digitales (págs. 11-28). Egregius Ediciones.

Durán, S., García, J. y Parra, M. (2017). Diversidad cultural y fomento de valores en la convivencia ciudadana. Revista busqueda, 4(19), 116-130. https://doi.org/doi. org/10.21892/01239813.366

Edwin, J. Santamaría, E., Moreno, K., y Santamaría, E. (2016). Aprovechamiento de la política pública en el fomento de la empresa familiar. Revista Retos, 6(11). https:// doi.org/10.17163/ret.n11.2016.02

Foglia, C. y Rofman, A. (2019). Gobernanza participativa local en el gran Buenos Aires: una radiografía actual de los 24 municipios. Revista Riem, (21), 113-145. http://dx.doi.org/10.4067/S071917902020000100113

Fung, A. (2015). Putting the public back into governance: The challenges of citizen participation and its future. Public Administration Review, 75(4), 513-522. https://onlinelibrary.wiley. com/doi/abs/10.1111/puar.12361

Fuster, D. (2019). Investigación cualitativa. método fenomenológico hermenéutico. Propósitos y Representaciones, 7(1), 201-229. https://doi.org/10.20511/pyr2019. v7n1.2678

García, I. M. (2007). La nueva gestión pública: evolución y tendencias. Presupuesto y Gasto Público, (47), 37-64. https://bit.ly/3ycnV3X

Garcia-Castillo, N., Bueno-Doral, T. y Liisa-Irene, H. (2020). Investigación e innovación respnsables (RRI) com motor de cambio en la comunicación corporativa: nuevas formas de gobernanza y estructuras participativas. Revista el prpfesioanl de la investigación, 29(3). https:// 
Palacios Garay, Jessica Paola; Toledo-Córdova, Marianela Francisca; Miranda-Aburto, Elder Jaime; Flores Farro, Angélica

Políticas públicas para una gobernanza participativa

\section{recyt.fecyt.es/index.php/EPI/article/} view/72911/50059

González-Monteagudo, J. (2001). El paradigma interpretativo en la investigación social y educativa: nuevas respuestas para viejos interrogantes. Cuestiones pedagógicas, (15), 227-246. https:// idus.us.es/handle/11441/12862

Hernández-Sampieri, R. y Mendoza, C. (2018). Metodología de la investigación, Las rutas cuantitativas, cualitativas y mixta. Mcgraw-Hill Interamericana editores S.A.

Jaime, F. M., Dufour, G. A., Alessandro, M. y Amaya, P. (2013). Introducción al análisis de políticas públicas. Universidad Nacional Arturo Jauretche. http://biblioteca. clacso.edu.ar/Argentina/icsyaunaj/20171114040327/pdf 1260.pdf

León, A. y Figueroa, S. (2019). Deliberación democrática y gobernanza en la participación ciudadana local. El caso de los consejos comunales de la sociedas civil (cosoc), Biobío, Chile. Revista Desafios, 32(2), 1-38. https://doi. org/10.12804/revistas.urosario.edu. co/desafios/a.7219

Ley de Bases de la Descentralización (2002). 27783/2002, de 17 de julio. https://bit.ly/3qzVsll

Ley de los Derechos de Participación y Control Ciudadanos (1994). 26300/1994, de 18 de abril. https:// pdba.georgetown.edu/Electoral/ Peru/participycontrol.pdf

Ley de Reforma Constitucional (2002). 27680/2002, de 7 de marzo. Diario Oficial El Peruano. https://bit. Iy/3helxCK

Ley de Transferencia y Acceso a la Información (2002). 27806/2002, de 2 de Agosto. https://www.peru.gob. pe/normas/docs/ley 27806.pdf

Ley del Procedimiento Administrativo General (2001). 27444/2001, de 10 de abril. http://www.pcm.gob.pe/ wp-content/uploads/2013/09/Leyde-Procedimiento-Administrativo-dePersonalLey27444.pdf

Ley Marco de Modernización de la Gestión del Estado (2002). 27658/2002, de 17 de enero. http://www4.congreso.gob. pe/comisiones/2002/modernizacion/ leyes/27658.htm

Ley Orgánica de Municipalidades (2003). 27972/2003, de 27 de mayo. https:// bit.ly/2UdnlUy

Martínez, M. (2006). La investigación cualitativa (síntesis conceptual). IIPSI, Revista de INvetaigaciónn y Psicología. Facultad de Psicología UNMSM, 123-146.

McLaverty, P. (2017). Public participation and innovations in community governance. Routledge.

Mora Belandria, E. G. (2002). Las políticas públicas y la necesidad de una verdadera política social en Venezuela. Revista De Ciencias Sociales, 8(3). https://doi. org/10.31876/rcs.v8i3.25189

Peña, J. y Bayardo, M. (2016). Consideraciones metodológicas en el estudio de la formación para la investigación de un marco interpretativo fenomenológico hermenéutico. Educación y Ciencia, $5,94-104$

Pérez, A. B. (2019). Metodología, métodos, técnicas. Propuesta metodológica para valorar políticas públicas. Estudios sobre las Culturas Contemporáneas Época III, 25 (5), 183-207.

Pinto, T., Martins, S. y Leonel, D. (2018). Gobernanza participativa: posibilidades y desafios en la gestión 
local. Revista ineteracoes (Campo Grande), 19(3), 627-641. https://doi. org/10.20435/inter.v19i3.1730.

Pulgarín, A., Bustamante, H. y Zapata, L. (2019). Noción de interés público en las constituciones de Iberoamerica. Revista contaduría universidad de Antioquia, (75), 13-42. https://doi. org/10.17533/udea.rc.n75a01

Quintero, C. (2017). Gobernanza y teoría de las organizaciones. Revista perfiles latinoamericanos, 25(50), 3957. https://doi.org/10.18504/pl2550$\underline{003-2017}$

Quinteros, J. A. (2020). Políticas públicas con enfoque en derechos humanos. Caleidoscopio - Revista Semestral de Ciencias Sociales y Humanidades, 24(43). https://doi. org/10.33064/43crscsh1518

Retamozo, M. (2009). Las demandas sociales y el estudio de los movimientos sociales. Revista cinta de moebio, (35). http:// dx.doi.org/10.4067/s0717 $\underline{554 \times 2009000200003}$

Reyes, F. \& Ortiz, H. (2017). Gestión pública y ciudadanía: Una reflexión en torno a las teorías sobre la democracia. Noveno Congreso Latinoamericano de Ciencia Política (ALACIP). Montevideo, Uruguay. https://bit.ly/3qzLr8h

Rodríguez, J. (2017). Políticas públicas. Revista venezolana de enfermería, 4(2), 17-26. http://190.169.30.98/ ojs/index.php/rev venf/article/ view/15917

Sánchez, J. (2015). La participación ciudadana como instrumento del gobierno abierto. Revista espacios públicos, 18(43), 51-73. https://www. redalyc.org/pdf/676/67642415003. pdf

Subirats, J. (2012). Democracia, participación y transformación social. Revista latinoamericana, Polis. https://journals.openedition.org/ polis/5599

Traba, L., Barletta, M.\& Velasquez, J. (2020). Teoría y práctica de las organizaciones herramientas para la gestión de la calidad. Santa Fé, Argentina. https://bibliotecavirtual. unl.edu.ar:8443/bitstream/ handle/11185/5584/Traba web. pdf? sequence $=1$ \&isAllowed $=y$

Vásquez, J.E. (2016). Revisión Teórica de las políticas públicas para determinar componentes iniciales de un modelo para la planeación de la contratación del departamento de Antioquia. Estudios de Derecho, 73(162), 77-105. https://dialnet.unirioja.es/ servlet/articulo?codigo $=6766589$

Vinasco-Hernández, J. (2019). Gobernanza y participación: la lucha por el territorio y las acciones comunitarias desde las metodologías participativas en la vereda Granizal, municipio de Bello. Revista equidad y desarrollo, 1(34), 79-100. https://doi. org/10.19052/eq.vol1.iss34.4

Wilson, J. (2018). ¿Qué son y para qué sirven las políticas públicas? Revista IN IURE, 2(8), 30-41. https:// revistaelectronica.unlar.edu.ar/index. php/iniure/article/view/492/436 